\title{
Phosphorus Recovery from Excess Sludge: Possibility and Future
}

\author{
Ming GAO ${ }^{1, a}$, Liping QIU ${ }^{1, b *}$, Kang XIE ${ }^{1, c}$, Shoubin ZHANG $^{1, d}$, Jiabin WANG ${ }^{1, e}$ \\ ${ }^{1}$ School of Civil Engineering and Architecture, University of Jinan, No.336.West Road of Nan Xin \\ zhuang. Jinan. 250022, PR China \\ a'Gaoming2717@163.com, ${ }^{b}$ lipingqiu@163.com, ${ }^{\mathrm{c}}$ cea_xiek@ujn.edu.cn, ${ }^{\mathrm{d}} \mathrm{cea} z$ zhangsb@ujn.edu.cn, \\ ecea_wangjb@ujn.edu.cn \\ ${ }^{*}$ Corresponding Author
}

Keywords : sewage sludge; phosphorus resources; phosphorus release; phosphorus recovery

\begin{abstract}
For the sake of the shortage of phosphorus resources and the present situation of municipal sludge treatment and disposal, the necessity of recovery of phosphorus from municipal sludge is analyzed, whereas the methods of phosphorus releasing from solid phase to liquid phase in sewage sludge, such as anaerobic digestion, ozone oxidation, heat treatment, ultrasonic dissolution and alkaline hydrolysis, were discussed in this paper. And then the theories and methods of phosphorus recovering from sewage sludge were summarized, including chemical precipitation, adsorption desorption, burning heat treatment and nanofiltration. Finally, the research development and application prospects of phosphorus release - recovery technology from sewage sludge were also proposed.
\end{abstract}

\section{Introduction}

According to statistics, phosphate has been listed as China in 2010 after the national economy, cannot meet the needs of the development of one of the 20 minerals. By the end of 2014, the amount of sludge produced by China years (by $80 \%$ water content) of about 30 million tons, annual sludge production (at $80 \%$ moisture content) will increase to 246 thousand $\mathrm{m}^{3}$.Currently in the process of sewage treatment, phosphorus in the form of residual sludge in the final discharge, the phosphorus content of about 4\% 9\%.But the sludge treatment and disposal technology in current hardly realize recovery of phosphorus resources. Therefore, it open up a new direction for the municipal wastewater treatment plant sludge treatment and disposal by using the appropriate technology to recover phosphorus from municipal sewage sludge. This paper summarizes the research status of phosphorus recovery and utilization in sludge, and prospects the research, development and application prospect of phosphorus release and recovery technology in sludge.

\section{Release of phosphorus in sludge}

Stable and efficient phosphorus release method is the most important prerequisite for the recovery of residual sludge. At present, the research on phosphorus release is mainly focused on the following aspects.

Anaerobic digestion.Sludge anaerobic digestion to sludge in anaerobic conditions, by facultative and anaerobic bacteria will sludge can be biodegradable organic matter decomposition into carbon dioxide, methane, and water, which is one of the common means of sludge reduction, stabilization.Bi Dongsu ${ }^{[1]}$ studied on the anaerobic digestion process of phosphorus rich excess sludge found that the phosphorus content of residual sludge 
supernatant increased from $0.90 \mathrm{mg} / \mathrm{L}$ to $102.90 \mathrm{mg} / \mathrm{L}$ after $18 \mathrm{~d}$ anaerobic digestion at room temperature. The method of anaerobic digestion is used to release the phosphorus in the sludge, which has the advantages of simple operation, obvious effect of phosphorus release, stable operation and low cost. At the same time, it also has a long reaction time, large reactor volume, and the shortcomings of the initial investment.

Ozone oxidation method.Ozone oxidation method is by ozone cytolysis, not easy to destroy biological degradation of the cell membrane, so that the intracellular substances can quickly dissolved in the liquid phase, so not only can realize the reduction of sludge and can promote the release of phosphorus in the bacterial cell. Ozone oxidation method has the advantages of ozone oxidation ability strong, good sludge disintegration effect, plant covers an area of less; the disadvantage is that sludge disintegration depends on ability to ozone input ratio, in the large-scale application of high investment ratio will lead to a high level of energy consumption.

Heat treatment method.Heat treatment method is the use of external heat source in the disintegration of sludge floc structure, the sludge in a part of cell material never dissolved into dissolved phosphorus, so that the more rapid release ${ }^{[2]}$.Kuroda ${ }^{[3]}$ et al. Akio showed that the concentration of $3500 \mathrm{mg} / \mathrm{L}$ activated sludge, after heating $60 \mathrm{~min}$ at $70 \square$, the total phosphorus release concentration of sludge reached $110 \mathrm{mg} / \mathrm{L}$, and the higher the temperature, the higher the rate of phosphorus release. The heat treatment method has the advantages of good phosphorus releasing effect and short reaction time, and the disadvantage is that the energy consumption is large in the heating process.

Ultrasonic method.Ultrasonic lysis method is by means of ultrasonic effect in the liquid produced a series of physical and chemical reaction, change the characteristics of the liquid in the dissolved and particulate substances, destruction of cell structure in sludge, the release of the organic matter, nitrogen, phosphorus and other elements from the solid state method. The reaction condition is mild, the reaction time is short, the effect of sludge is good, but the energy consumption is large, and the strength and reaction time still need to be optimized in order to be applied in large scale.

Alkali solution.The alkaline treatment of sludge by adding alkali to promote the dissolution of fiber content in the sludge, alkaline conditions can effectively inhibit the activity of activated sludge, intracellular material dissolution of the crack technology. Xiao Benyi and other ${ }^{[4]}$ compared the effects of acid hydrolysis, alkali hydrolysis and pyrolysis of 3 kinds of sludge treatment methods on the nature of the sludge, found that the effect of alkali solution is best, the cracking rate of sludge reached $58.46 \%$. Compared with other methods, the alkaline hydrolysis method has the advantages of simple equipment, easy operation and high efficiency. The PH of the sludge supernatant is alkaline, which provides a basic environment for phosphorus crystallization. But the reaction consumes a large amount of alkali, high investment .

\section{Method for recovering phosphorus from sludge}

At present, the main recovery methods of phosphorus rich supernatant are physical and chemical methods such as chemical precipitation method and other recovery methods.

Magnesium ammonium phosphate crystallization method.In the process of residual sludge disintegration, the nitrogen element is usually accompanied with the release of nitrogen, and the nitrogen and phosphorus in the sludge can be recovered by the MAP method. Struvite is rich in nitrogen, phosphorus content, is a direct application of the farmland slow-release fertilizer; Wang et al. [6] found that map crystallization of the optimal $\mathrm{pH}$ was 9.0. Moerman ${ }^{[7]}$ found that calcium ion interference effect in the process of formation of struvite. Generally considered to maintain $\mathrm{Mg}^{2+}: \mathrm{PO}_{4}{ }^{3-}=1.3: 1.0$ is more appropriate. According to the above conditions, the recovery of phosphorus in the residual sludge hydrolysis acidification solution can be recovered by MAP

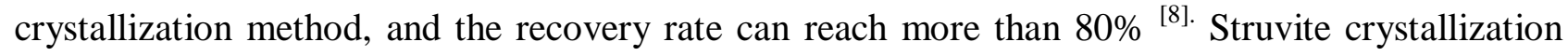
into medicament price is high, due to the limitation of the purity, recovery of struvite economic 
value has not been fully developed, and so the method in the reaction conditions and the purity of the products still need to further study.

Calcium phosphate compound precipitation method.In the appropriate chemical environment, different types of calcium phosphate compounds will be precipitated from the solution, among them, $\mathrm{Ca}_{5}$ $\left(\mathrm{PO}_{4}\right)_{3} \mathrm{OH}$ (that is, hydroxyapatite, HAP) is the most stable. HAP In the reaction process, $\mathrm{PH}$ is generally controlled at more than 9.2 , while the $\mathrm{Ca} / \mathrm{P}$ ratio is above 2 , and the recovery of phosphorus can reach more than $90 \%{ }^{[9]}$. The method has the characteristics of low cost, high recovery efficiency. However, the $\mathrm{Ca}(\mathrm{OH})_{2}$ dosage of the reaction is larger, and the economic value of the calcium phosphate is low. Therefore, the method needs to be optimized in the actual production.

Other phosphorus recovery technologies.Adsorption analytical method.Adsorption / analytical method is a physical and chemical method to recover phosphorus from phosphorus rich solution. Adsorption and desorption method of phosphorus adsorption recovery efficiency is high, but the adsorption agent often need after treatment in acidic or alkaline conditions, in order to achieve good recovery rate of phosphorus and a certain amount of acid or alkali consumption is needed in the process of analysis, which in the economy to the technique adds a lot of obstacles.

Nano filtration. Using Nano filtration to divalent or multivalent ions and molecular weight between 200-1000 between organic matters have characteristics of high removal rate of phosphorus recovery. Christoph et al Blocher[10] using Nano filtration technology will be after the treatment of sludge supernatant was between phosphorus and heavy metals separation and recovery of the $54 \%$ of phosphorus, but it is still in the experimental stage, need to be further solved blockages in the low recovery rate and reduce membrane pollution problems.

\section{Conclusions}

In a summary, it is feasible and advantageous by using the alkaline hydrolysis method to crack and release the phosphorus from the exceed sludge and then recovering by MAP and HAP, with the characteristics of high recovery efficiency. In future studies on phosphorus recovery process, it should been pay more research attention to all kinds of novel technologies, not only for the sludge treatment and disposal but also the phosphorus recovery, in order to solve the global problem of phosphorus resource shortage.

\section{Acknowledgements}

This study was partly supported by National Natural Science Foundation of China (51278225), Science and technology development projects of Shandong province (2013GSF11704), Natural Science Foundation of Shandong province (ZR2013EEQ007, ZR2015EM021), and Science and technology development projects of Jinan (201302079).

\section{References}

[1]Dong.Su.Bi.,Xiao.Pin.Guo.Journal of Environmental Sciences , VOL30. (2010) , p. 2446-2449."In Chinese”

[2]Ben.Yi.Xiao , Hong.Yan , Journal of Environmental Sciences , VOL29.(2009) , p.673-682.“In 
Chinese"

[3]Akio Kuroda , Noboru Takiguchi , et al: Biotechnology and Bioengineering , VOL78.(2002), p. 333-338.

[4]Ben.Yi.Xiao , Jun.Xin.Liu, Journal of Environmental Sciences, VOL29. (2008), p.327-331.“In Chinese"

[5]J.Wang, Y.Song,P.Yuan , et al: Chemosphere , VOL65.(2006), p. 1182-1187.[6]W.Moerman,M.Carballab,IWA Publishing ， VOL(2009),p245-256.

[7]Juan.Tong , Guang.Yin.Chen.Techniques and Equipment for Environmental Pollution Control , VOL1.(2007).p.1-5."In Chinese”

[8]Zhen.Min.Chen „Song.Yuan.Wei „Jun.Xin.Liu,et al: China Water \& Wastewater ,VOL25.(2009) , p. 22-25."In Chinese"

[9]C.Bloher , C. Niewersch , T. Melin. Water Research , VOL46.(2012), p.2009-2019. 